\title{
Permanent, Non-Leaching Antimicrobial Polyamide Nanocomposites Based on Organoclays Modified with a Cationic Polymer
}

\author{
Rinat Nigmatullin, ${ }^{*}$ Fengge Gao, Victoria Konovalova
}

The intercalation of cationic copolymer into a smectic clay, montmorillonite, has been used to produce polymerically modified organoclays. The organoclays of different lamellar morphology and content of quaternary ammonium groups have been prepared by altering the clay/polymer ratio. The organoclays prepared have been explored in the design of antimicrobial materials based on clay/polymer nanotechnology. Polyamide nanocomposites containing organoclays with incorporated cationic polymer showed an antimicrobial activity and improved mechanical properties. The antimicrobial efficiency and the mechanical properties of the nanocomposites were controlled by the variation of the content of the cationic polymer incorporated into the organoclay and organoclay loading.

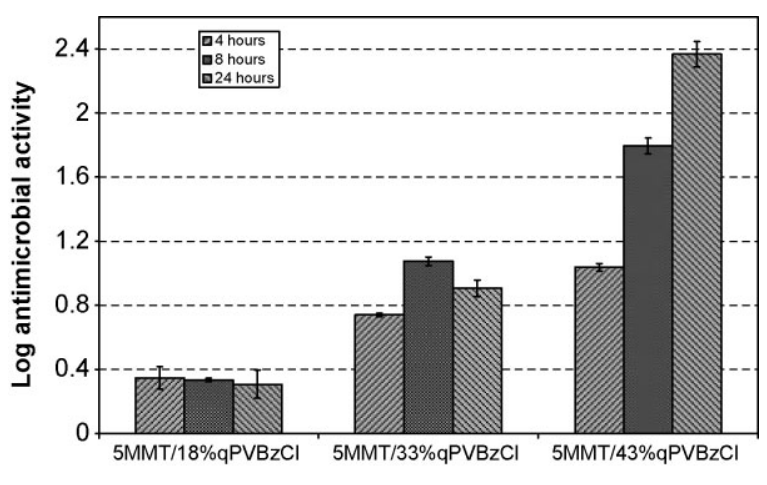

\section{Introduction}

The increase in numbers of antibiotic-resistant microbes and related disease outbreaks, the declination of immunity due to population aging and environment pollution, the increase of global human and livestock mobility, require extensive activities in infection control. The old and current practice to combat infections mainly relies on disinfection methods that aim to inhibit the microbial growth on surfaces via special chemical or physical treatments. The disinfectant migration from the surface and the loss of its activity due to various interactions ultimately result in unprotected surfaces.

R. Nigmatullin, F. Gao

School of Science and Technology, Nottingham Trent University, Clifton Lane, Nottingham NG11 8NS, UK

Fax: +44 115848 6636; E-mail: rinat.nigmatullin@ntu.ac.uk

V. Konovalova

Department of Chemistry, National University of Kyiv-Mohyla

Academy, 2 Skovoroda Street, Kiev 04070, Ukraine
Alternatively, biocide-containing materials have been used in various applications as self-sterilizing surfaces. ${ }^{[1-3]}$ The main approach in developing such materials is based on the entrapment of biocide substances into a matrix material. As a result, the material is able to release biocides and thereby to provide antimicrobial, antifungal or antiviral protection. However, biocide release rate decreases with time because of biocide depletion. Therefore, the surfaces can be protected only for a limited period of time. Moreover, the release of low-molecular-weight biocides into the environment, contributes to the development of microorganism resistance against biocides.

Binding biocide molecules onto the surface has the potential to overcome these problems if immobilized antimicrobial agents retain their activity and thereby impart antimicrobial properties to the surface. Recently, permanent rendering of surface biocidal activity (or, at least, for life-term of the material), has attracted growing interest as an alternative approach to suppress cell viability on solid surfaces. ${ }^{[3-5]}$ Significant efforts in developing surface-bound antimicrobials have been focused on 
quaternary onium salts. ${ }^{[5-11]}$ Cationic surfactants represent a broad range of organic disinfectants. ${ }^{[12]}$ In addition to lowmolecular-weight surfactants, cationic biocides have been further developed as polymer and copolymers containing quaternary onium groups. There are number of evidences that polymeric onium salts are more potent than their lowmolecular-weight counterparts in biocidal activities. ${ }^{[13]}$ The early development of polymeric biocides was focused on water or alcohol-soluble substances which could be used in a similar way to the low-molecular-weight surfactants. Over the past few decades, sporadic attempts were undertaken to explore antimicrobial activity of insoluble forms of the polymeric onium salts. ${ }^{[6-8,14-16]}$ More recently, research activities on self-sterilizing surfaces based on polymers containing onium groups have undergone a substantial growth. It is generally accepted that surfacebound polymers containing quaternary onium groups can suppress the viability of cells contacting the materials efficiently. There are strong evidences that the antimicrobial efficiency of a surface-bound polymer with onium groups is higher for the polymeric onium salts, which contain long alkyl chains in a quaternary group. ${ }^{[9,17]}$ The hydrophobic alkyl chains are believed to facilitate polymer penetration through bi-layered cell membrane, compromising its integrity and ultimately causing cell death. ${ }^{[5]}$

In the past two decades, long-chained quaternary onium salts have become a key element of thriving clay/polymer nanotechnology. ${ }^{[18,19]}$ Cationic surfactants are used to produce organophilic clays through the ion-exchange of the metal cations within the layered silicates. Conceptually, clay modification is intended to intercalate organophilic substances into the interlayer space in order to weaken the interlayer interactions, to increase the interlayer spacing and to improve clay-polymer compatibility. Such effects enable the penetration of macromolecules into the clay interlayer space during processing, leading to the separation of the individual silicate layers and the uniform dispersion of the separated layers in a polymer matrix. Composites produced in this way are filled with particles of nanometer scale and show the improvement of a wide range of physical and engineering properties at relatively low clay content, typically below 5 wt.-\%.

Despite nearly two decades of development of polymer/ clay nanotechnology, it was overlooked that organoclaybased nanocomposites actually contain antimicrobial entity, cationic surfactants, and have potential to be antimicrobial materials. Just recently, several research groups reported antimicrobial activity of clay/polymer nanocomposites based on commercially available organoclays. ${ }^{[20-26]}$ Although the migration of the surfactants from the nanocomposites has been experimentally confirmed, the antimicrobial action of the composites is believed to be dominated by solid surface activity on contact. ${ }^{[20]}$ Surfactant migration from the nanocomposites produced from those commercially available organoclays, not only restricts materials applications, but also can eventually result in the loss of the antimicrobial activity due to biocide exhaustion.

A novel solution has been developed in this study to prevent the migration of antimicrobial modifiers towards developing non-leaching antimicrobial materials via clay/ polymer nanotechnology. The strategy applied in this research is to use macromolecular substances containing quaternary onium groups for clay modification. We assume the formation of the multiple ionic bonds between the multi-charged modifiers and one or several clay platelets during the ion exchange. The cooperative interactions are expected to exclude unbound state of the modifier and thereby to prevent polymeric biocide migration from both the organoclays and nanocomposites.

The ability of cationic copolymers to intercalate mineral clays has been proven for several polymer modifiers such as end-capped polymers, ionenes, branched polymers, graft-, block- and random copolymers. ${ }^{[27]}$ The term polymerically modified organoclays was previously used to refer to organoclays with chemically bound polymers. This definition allows distinguishing such materials from the intercalated structures when a polymeric intercalant is not chemically bound to the clay surface. Additionally, polymer-modified and macromolecule-modified organoclays will be used in this paper as equivalents of polymerically modified organoclays. Similar to organoclays with the incorporated low-molecular-weight organic substances, the polymerically modified clays can facilitate the dispersion of clay in a polymer matrix to form nanocomposites. Moreover, compared with cationic surfactants, it is anticipated that copolymers could offer more flexibility in tailoring modifier properties as a compatibilizer between the clay and a targeted polymer matrix.

In this study, poly(vinylbenzyl chloride) (PVBzCl) partially aminated with a tertiary amine containing one long alkyl chain, has been used as an example of antimicrobial macromolecular clay modifier in the development of nonleaching polymer nanocomposites, which are effective against microorganisms by contact. Organoclays with different content of the antimicrobial polymer modifier have been prepared and used to produce polyamide 6 nanocomposites. The antimicrobial activity of the nanocomposites was investigated against Escherichia coli and Staphylococcus aureus strains. The influences of organoclay composition and content on antimicrobial activity, mechanical properties and morphology of the nanocomposites have been studied.

\section{Experimental Part}

\section{Materials}

Montmorillonite (MMT) (sodium form) with cation exchange capacity of 0.92 mequiv. $\mathrm{g}^{-1}$ was supplied by the Southern Clay 
Products. PVBzCl with molecular weight $55 \mathrm{kDa}$ was purchased from the Scientific Polymer Products (Ontario, NY, USA). N, Ndimethylhexadecylamine and tetrahydrofuran (THF) were supplied by Sigma-Aldrich (Loughborough, UK) and were used without further purification. The polyamide- 6 studied was Ultramid ${ }^{\mathbb{R}}$ B3 purchased from BASF.

\section{Synthesis of Partially Aminated Poly(vinylbenzyl chloride)}

The synthesis of a derivatized $\mathrm{PVBzCl}$ containing quaternary ammonium groups ( $\mathrm{qPVBzCl}$ ) was carried out by partial amination of the methylene chloride groups with a tertiary amine (Scheme 1). In a round-bottom flask equipped with a reverse condenser, $40 \mathrm{~g}$ of $\mathrm{PVBzCl}$ were dissolved in $500 \mathrm{~mL}$ of THF to produce a polymer solution. $26.5 \mathrm{~mL}(78.6 \mathrm{mmol})$ of $N, N$-dimethylhexadecylamine were added to the polymer solution resulting in 3:1 molar ratio of vinylbenzyl chloride units to tertiary amine. The reaction in the mixture was carried out at $60^{\circ} \mathrm{C}$ for $24 \mathrm{~h}$ under constant stirring. After the reaction, portions of $\mathrm{qPVBzCl}$ polymer solution were used directly for organoclay preparation.

\section{Preparation of Polymerically Modified Organoclays}

Sodium-montmorillonite (Na-MMT, $8 \mathrm{~g}$ ) was vigorously stirred in $400 \mathrm{~mL}$ of distilled water using a magnetic bar in order to produce a clay suspension. The suspension was stirred at ambient temperature overnight. A portion of qPVBzCl solution was diluted by $200 \mathrm{~mL}$ of THF. Clay suspension was added to the diluted polymer solution with vigorous stirring. Copious precipitates were formed immediately after clay introduction. A further $50 \mathrm{~mL}$ of water was used to insure the complete MMT transfer into the reaction mixture. The slurry was stirred at ambient temperature for $24 \mathrm{~h}$ and followed by repeated centrifugation and washing with 50:50 water/THF mixture and distilled water three times with each solvent system. After the final washing and centrifugation, the organoclays containing the polymer modifier were dried using freeze drying. Three types of organoclays were prepared using 25, 40 and $55 \mathrm{~g}$ portions of $\mathrm{qPVBzCl}$ solutions. The content of the polymeric modifier in the organoclays was determined by thermal gravimetric analysis (TGA) and shown in the Table 1 with the corresponding material coding. TGA was performed on a PerkinElmer Pyris 1 TGA by increasing temperature from $40-800^{\circ} \mathrm{C}$ at a rate of $10^{\circ} \mathrm{C} \cdot \mathrm{min}^{-1}$ under a constant nitrogen flow of $20 \mathrm{~mL} \cdot \mathrm{min}^{-1}$. The content of polymer modifier was determined as weight loss during sample heating from $120^{\circ} \mathrm{C}$ (after water evaporation) and $800^{\circ} \mathrm{C}$.

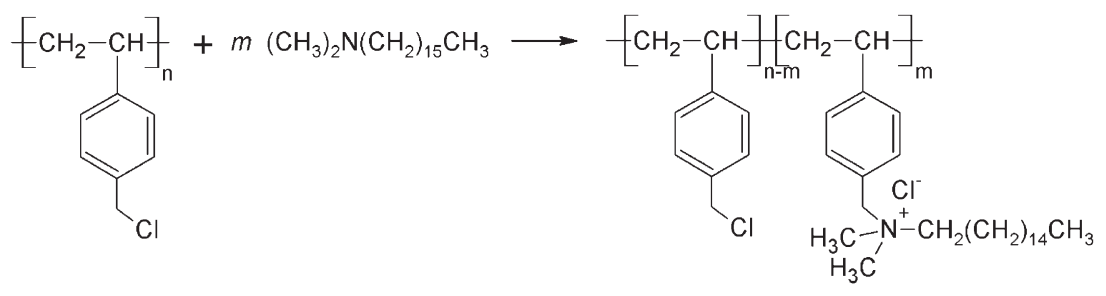

Scheme 1.
Table 1. Composition and coding of the prepared materials.

\begin{tabular}{|c|c|c|c|c|}
\hline \multirow[t]{2}{*}{ Clay } & \multirow{2}{*}{$\frac{\text { MMT }}{\text { wt.-\% }}$} & \multirow{2}{*}{$\frac{q P V B z C l}{\text { wt.-\% }}$} & \multirow{2}{*}{$\begin{array}{c}\text { Polyamide } 6 \\
\text { nanocomposite }\end{array}$} & \multirow{2}{*}{$\frac{\text { Clay }^{\mathrm{a}}}{\text { wt.-\% }}$} \\
\hline & & & & \\
\hline $18 \mathrm{qPVBzCl}$ & 82 & 18 & 5MMT/18q PVBzCl & 5 \\
\hline \multirow[t]{3}{*}{$33 \mathrm{qPVBzCl}$} & 67 & 33 & 2.25MMT/33qPVBzCl & 2.25 \\
\hline & & & 4MMT/33qPVBzCl & 4 \\
\hline & & & $5 \mathrm{MMT} / 33 \mathrm{qPVBzCl}$ & 5 \\
\hline $43 q \mathrm{PVBzCl}$ & 57 & 43 & $5 \mathrm{MMT} / 43 \mathrm{qPVBzCl}$ & 5 \\
\hline
\end{tabular}

a) Based on unmodified MMT.

\section{Nanocomposite Preparation}

Polyamide/clay nanocomposites were prepared by melt extrusion using a $16 \mathrm{~mm}$ twin-screw extruder. The extrusion was carried out under the condition of temperature $240^{\circ} \mathrm{C}, \mathrm{L} / \mathrm{D}$ (length/diameter) ratio $24: 1$, screw speed $400 \mathrm{rpm}$ and feeding rate $20 \%$. Two series of nanocomposites were produced using pre-dried and pre-mixed formulations of polyamide/clay. One series contained $5 \mathrm{wt} . \%$ of each type of polymerically modified organoclay prepared (18qPVBzCl, 33qPVBzCl and 43qPVBzCl). The second series was produced using the organoclay with $33 \mathrm{wt} . \%$ of $\mathrm{qPVBzCl}$ and contained 2.25, 4 and 5 wt.-\% of the organoclay. The composition of the polyamide nanocomposites and their corresponding codes are shown in Table 1. The extruded strands were pelletized and dried in a vacuum oven at $80^{\circ} \mathrm{C}$ for $10 \mathrm{~h}$ before molding into bone-shaped coupons $\left(5 \times 3 \mathrm{~mm}^{2}\right)$ for tensile test, bars $\left(9 \times 3 \times 50 \mathrm{~mm}^{3}\right)$ for dynamic mechanical analysis (DMA) or square samples $(25 \times 25 \times$ $1 \mathrm{~mm}^{3}$ ) for antimicrobial and XRD tests using injection molding. The temperature applied in the injection molding was $270^{\circ} \mathrm{C}$.

\section{XRD Characterization of Organoclays and Nanocomposites}

The X-ray diffraction (XRD) characterization of the organoclays and nanocomposites was carried out using a Philips XPert Pro XRD (PANalytical, USA) with $\mathrm{Cu} \mathrm{K}_{\alpha}$ radiation source at $40 \mathrm{kV}$ and $30 \mathrm{~mA}$. The samples were scanned at a scan rate of 1 degree per minute with the step size of 0.008 degrees.

\section{Mechanical Tests of Nanocomposites}

The tensile strength of the nanocomposites was measured according to ASTM D638 using a Monsanto tensiometer with a cross-head speed of $0.25 \mathrm{~mm} \cdot \mathrm{s}^{-1}$. Since the limitation of sample size, it was difficult to fit strain gauge or extensometer to the sample during tensile loading. Therefore only strength data was reliable. The stiffness and other mechanical properties were studied using dynamic mechanical testing. The storage modulus $\left(G^{\prime}\right)$, 
loss modulus $\left(G^{\prime \prime}\right)$ and the ratio of $G^{\prime \prime}$ and $G^{\prime}$, i.e., $\tan \delta$, were measured using a Bohlin C-VOR torque dynamic mechanical tester (Malvern Instruments, Malvern, UK). The measurements were performed in air with heating rate $2{ }^{\circ} \mathrm{C} \cdot \mathrm{min}^{-1}$ in stress-controlled mode with shear stress of $0.455 \mathrm{MPa}$. A program was applied to heat the material starting from 25 to $140^{\circ} \mathrm{C}$ with an oscillation frequency of $1 \mathrm{~Hz}$.

\section{Assessment of Antimicrobial Activity}

The antimicrobial activity of the nanocomposites was assessed according to the Japanese Industrial Standard Test Method JIS Z 2801: 2000 (Antimicrobial Product Test for Antimicrobial Activity and Efficacy). The method was developed specifically for the characterization of antimicrobial properties of solid surfaces including non-leaching materials. The antimicrobial activity was studied against two strains: gram-negative E. coli BE and grampositive $S$. aureus CCM 209 bacteria, which were received from the Ukrainian Collection of Microorganism. In brief, the antimicrobial tests of the prepared nanocomposites were carried out as following. A cell suspension of either $E$. coli or $S$. aureus with cell concentration of $2.5 \times 10^{5}-1.0 \times 10^{6}$ cells $\cdot \mathrm{mL}^{-1}$ was prepared in a $1 / 500$ nutrient broth. An aliquot $(150 \mu \mathrm{L})$ was placed onto at least 3 replicate subsamples of the nanocomposite and 3 replicate sub-samples of control surface (the pristine polyamide 6) and held in intimate contact using a sterile polyethylene film. The seeded nanocomposite samples and the control sub-samples were subsequently incubated for $24 \mathrm{~h}$ at $35^{\circ} \mathrm{C}$ at saturation humidity. After the incubation, the samples assembled with the polyethylene film cover were washed with nutrient broth. The number of colony forming units (CFUs) within the resulting suspensions was then enumerated using a pour plate method.

The antimicrobial activity of the nanocomposite material was calculated from the difference between the log 10 number of CFUs on the surface of the nanocomposite and the control surface according to the formula:

Antimicrobial activity $=\log (C / A)$

where $C$ and $A$ are the average number of viable cells of bacteria on the control test piece and on the antimicrobial test piece after $24 \mathrm{~h}$ respectively.

\section{Results and Discussion}

\section{Structure of Macromolecule-Intercalated Organoclays and Polyamide 6 Nanocomposites}

Similar to applying cationic surfactants, the clay modification by cationic polymers is based on ion exchange. In this study, the content of $\mathrm{qPVBzCl}$ in a partially aminated $\mathrm{PVBzCl}$ was adjusted to obtain a water insoluble qPVBzCl. Consequently, the clay modification must be conducted in organic solvents or aqueous organic mixture. Such reaction media are not favorable for clay modification due to the limitation of clay swelling and thereby accessibility of reactive functional groups on clay platelet, and the slower kinetics of ion-exchange reaction in organic media. Nevertheless, mixtures of some organic solvents with water such as water/THF, water/dioxane have been previously reported as suitable media to intercalate macromolecular cationic substances into the clay gallery. ${ }^{[27,28]}$

qPVBzCl is soluble in 50:50 water/THF mixture and such solvent mixture could be used to modify MMT. Mixing equal volumes of swollen MMT dispersed in water and the qPVBzCl solution in THF results in instantaneous copious formation of a polymer/clay precipitate. As can be seen from the XRD patterns in Figure 1(a), significant increase in interlayer spacing of the polymerically modified organoclays occurred with respect to Na-MMT. Thus the chosen
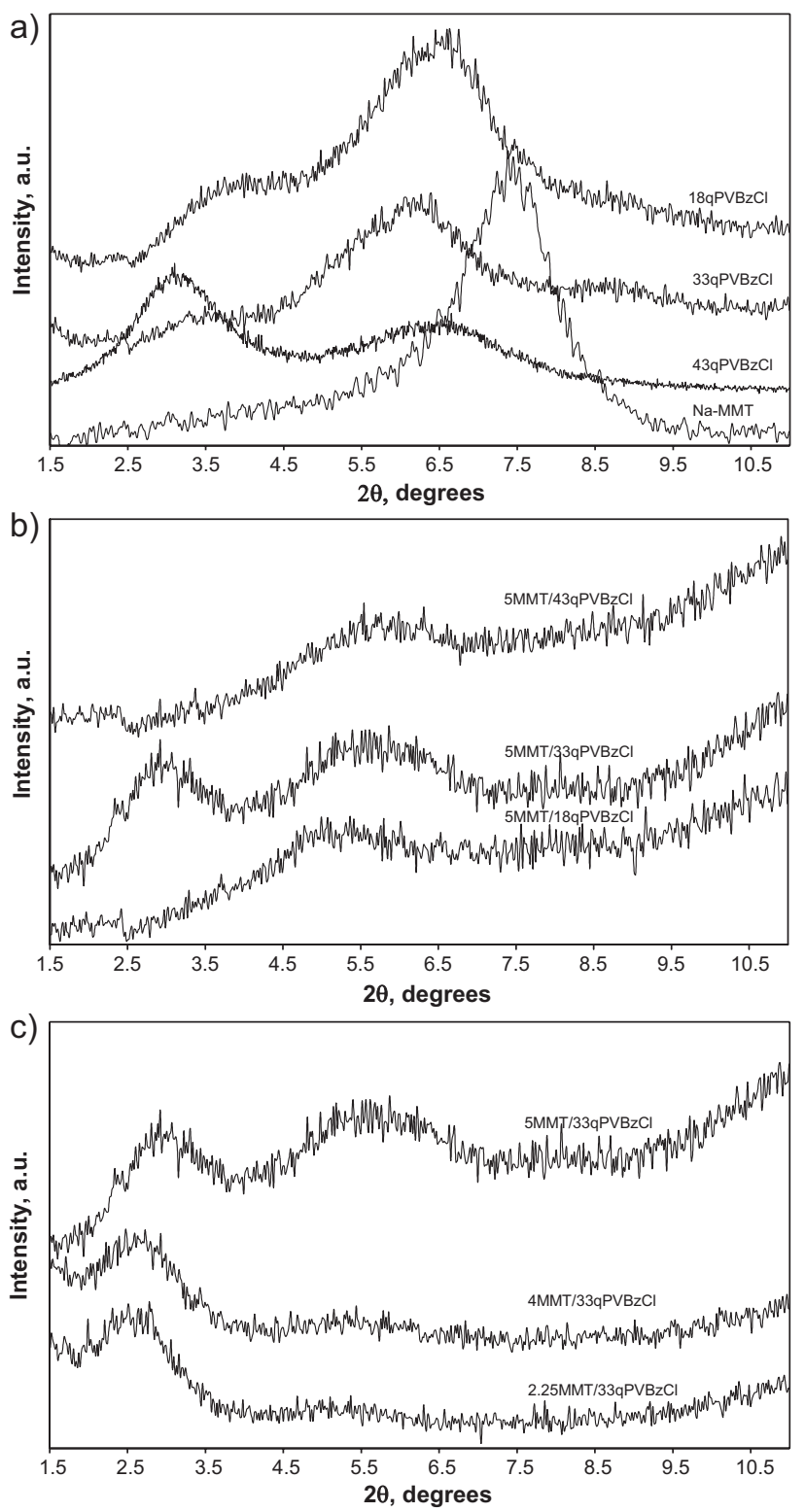

Figure 1. X-ray diffractograms of (a) clays and (b, c) polyamide 6 nanocomposites based on polymerically modified organoclays. 
solvent mixture and reaction conditions were suitable for the cation exchange reaction and the intercalation of cationic polymer into the interlamellar space of the clay.

An increase in polymer/clay ratio in the reaction mixture results in the variation of not only the quantity of $q \mathrm{PVBzCl}$ bound to clay but also the structure of the organoclay and their nanocomposites. The original Na-MMT has a single (001) peak at $2 \theta=7.54^{\circ}$ which corresponds to interlayer spacing of $1.17 \mathrm{~nm}$. Following the intercalation with different quantity of $\mathrm{qPVBzCl}$, two peaks in the region of $2 \theta$ below $7^{\circ}$ are visible for each organoclay. The ratio of the intensities of the two peaks is a function of $q \mathrm{PVBzCl}$ loading. At a lower loading level, the intensity of the peak at smaller $2 \theta$ angles is less than the peak at the larger angle. The relationship changes as the content of $\mathrm{qPVBzCl}$ in clay increases, the intensity at the smaller angle becoming higher and the intensity at the large angle lower. Apparently the peak at the larger angle is not the (002) peak since (002) peak should have much lower intensity than (001) peak. In our opinion, both peaks are (001) peak of the organoclay. In other words, different structures of organoclay have been formed. Similar XRD results have been reported previously for organoclays prepared by modification with both low $^{[29]}$ and high molecular weight $^{[30]}$ cationic substances. Such pattern of X-ray scattering was attributed to the formation of heterogeneous interlayer structures.

It appears that the intercalation of $\mathrm{qPBVzCl}$ causes nonuniform intercalation in the organoclay. As the content of a high molecular weight cationic polymer increases, the intercalated structure becomes more uniform. The evidence of this is that the peak at the larger angle disappears progressively with the increase in the $\mathrm{qPBVzCl}$ content. The peak at the smaller angle becomes progressively intensive and also shifted further to smaller angles. Organoclay containing $43 \mathrm{wt}$. $\%$ of cationic polymer showed higher order diffraction peak indicating the formation of uniformly structured material. This indicates that $\mathrm{qPBVzCl}$ is an effective intercalate and perhaps may even cause clay exfoliation when its loading increases further. However, it was not intended to use very high $\mathrm{qPBVzCl}$ loading in this study due to the consideration of its influence on physical and engineering properties of the final nanocomposites. The interlayer spacing of the organoclays prepared using $\mathrm{qPVBzCl}$ in the loading level applied, is comparable with the values reported for commercially available organoclays. ${ }^{[20]}$ This implies that the qPVBzCl-modified organoclays can facilitate dispersion of clay layers in a matrix of other polymers to produce nanocomposites.

For polymerically modified clays, the variation of polymer content not only changes the extent of layer expansion but also alters the content of functional groups, i.e., quaternary ammonium groups (QAGs) in the material. These groups are not necessarily bound to the clay surface, especially at higher contents of the polymeric modifier. It is anticipated that the higher content of OAGs would be beneficial to the antimicrobial activity of the material. On the other hand, the high OAG content can affect the compatibility of organoclay with the matrix polymer and consequently the extent of dispersion of clay layers within the polymer matrix. In order to investigate the influence of qPVBzCl on both layer dispersion of clay and antimicrobial activity, a series of polyamide 6 composites were produced varying organoclay content in the nanocomposites and qPBVzCl content for a fixed clay content. The XRD results for nanocomposites thus produced are shown in Figure 1(b) and (c).

As can be seen from Figure 1(b), for the polyamide 6 nanocomposites with 5 wt.-\% clay content, calculated based on the unmodified MMT, further layer expansion of clay occurred in each material. The (001) peak of wellintercalated structure, which corresponds to the peak at smaller angle in Figure 1(a), either disappeared or shifted further towards the smaller angle in Figure 1(b). The disappeared peaks may be shifted to the region beyond $2 \theta=1.5^{\circ}$. Part of the clay structure may be exfoliated in the polyamide. The organoclay peak at a larger angle [Figure 1(a)] shifted to a smaller angle for each nanocomposite material. This is the indication that polyamide 6 also participated in the intercalation and progressively filled the gallery space of the clay. The influence of $\mathrm{qPBVzCl}$ content in the clay on clay intercalation/exfoliation is complicated. The composite containing the clay with medium qPBVzCl loading level, i.e., 33\% qPBVzCl in the clay, results in the smallest extent of layer expansion. The reason for this has not yet been understood. Further investigation is on the way with the inclusion of other cationic polymer modifiers.

The effect of organoclay content on clay intercalation/ exfoliation in polyamide 6 was also studied. The XRD patterns of the composites containing different loading levels of the organoclay with 33 wt.-\% $\mathrm{qPBVzCl}$ in the clay are shown in Figure 1 (c). When the clay content is reduced from 5 to 4 and then to $2.25 \mathrm{wt}$. $\%$, only one (001) peak is visible in the XRD spectra for each material whilst two peaks are present for the composite containing $5 \mathrm{wt} . \%$ organoclay. The peak position of the composites containing 4 and 2.25 wt.-\% organoclay is identical and slightly lower than the peak at the smallest angle for the composite with the higher loading. It is not clear which organoclay peak in Figure 1 (a) are associated with the peak at $2 \theta=2.65^{\circ}$ for the two composites with lower loading in Figure 1 (c). It is possible that the well-filled part of the organoclay, which corresponds to the peak at the smaller angle, may have higher extent of exfoliation so that the original peak would not be visible. Meanwhile, the less expanded layers, which give the diffraction peak at the larger angle, have been further intercalated with polyamide. It is understandable 
that lower filler loading is always favorable to higher extent of exfoliation due to the reduced extent of particle and layer aggregation.

\section{Antimicrobial Activity of Polymer Nanocomposites}

For polymer/clay nanocomposites, it was estimated that overlapping of platelet of clay layers could occur when clay loading exceeded $1.2 \mathrm{wt.} . \% .{ }^{[31]}$ Therefore, even for relatively small clay loadings used in the polymer nanotechnology, clay platelets are practically omnipresent across the surface of the nanocomposite material. As a result, clay platelets functionalized with some modifiers can impart effectively the composite surface with these functionalities. Alkyl dimethyl benzyl ammonium salts, which are low-molecular-weight analogues of $\mathrm{qPVBzCl}$, are wellknown biocides ${ }^{[12]}$ and have shown the antimicrobial activity in the bound state. ${ }^{[32]}$

It is noteworthy to mention that the method for the antimicrobial tests must consider the potential applications of the materials and the mode of the antimicrobial action. ${ }^{[4]}$ The nanocomposites developed in this study together with many surface modified materials described in literature are expected to act by contact without leaching biocides. As a result, biocides would not be present in a liquid media, for example, bacterial suspension, after contact with such materials. Accordingly, testing the antimicrobial activity using 'shaking flask' or inhibition zone methods are practically meaningless for non-leaching type antimicrobial materials. The design of material active by contact, is aimed to cope with microorganisms deposited on the surface, most likely either in low nutrient media or at solid/air interface. Therefore, the antimicrobial activity of such materials has to be evaluated, based on the viability of microorganisms kept in close contact with the material, for example, deposited or adhered microorganisms. The cell viability could be measured by monitoring bacterial growth on the surface covered with a solid nutrient media ${ }^{[9]}$ or by viable cell counting in a bacterial suspension produced by re-suspension of cell adhered to the material surface. The latter approach has been developed into the Japanese Industrial Standard JIS Z 2801: 2000 and is suitable for studying antimicrobial activity of polymer nanocomposites.

Figure 2 shows the antimicrobial activity of a series of nanocomposites with different loading levels of organoclay containing $33 \mathrm{wt} . \%$ of $\mathrm{qPVBzCl}$ against S. aureus. Log reduction in the number of viable cells increases from 0.4 to 1.4 with the increase in organoclay content from 2.25 to 5 wt.-\%. Even the nanocomposite with the lowest organoclay content can significantly decrease the number of viable cells adhered to the material surface. This confirms that cationic polymer bound to clay layer preserves its antimicrobial activity following clay dispersion in the

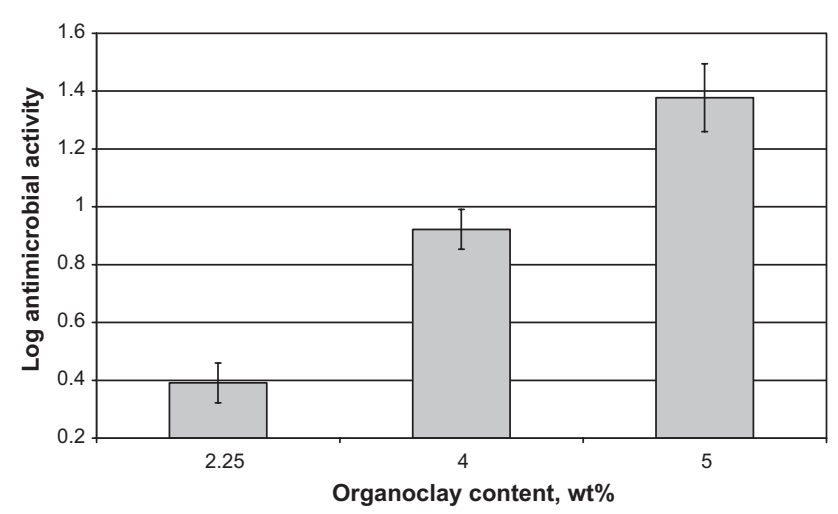

Figure 2. The influence of the content of polymerically modified organoclay on the antimicrobial activity of polyamide 6 nanocomposites based on organoclay containing $33 \mathrm{wt} .-\% \mathrm{qPVBzCl}$.

polyamide matrix. The antimicrobial activity improves with the increase of organoclay content in the nanocomposites. Although higher contents of organoclay in the composite materials tends to provide the better antimicrobial activity of the nanocomposites, the improvement of the antimicrobial activity is not a simple reflection of the change in the quantity of the antimicrobial agent. Organoclays are intercalated structures with cationic polymer located within the clay galleries. In order to exhibit antimicrobial activity by contact, cationic polymer must be exposed to the material surface. Therefore, observed antimicrobial activity of the nanocomposite surface gives further evidence of clay layers delamination to a certain extent during the melt extrusion process.

Similarly, for nanocomposites with the same clay loading, the content of the antimicrobial agent can be changed by varying the amount of bond cationic polymer in the organoclay. Figure 3 shows the antimicrobial activity of these nanocomposites containing the same content of organoclay but different content of $\mathrm{qPVBzCl}$ in the organoclay against gram-positive $S$. aureus and gram-

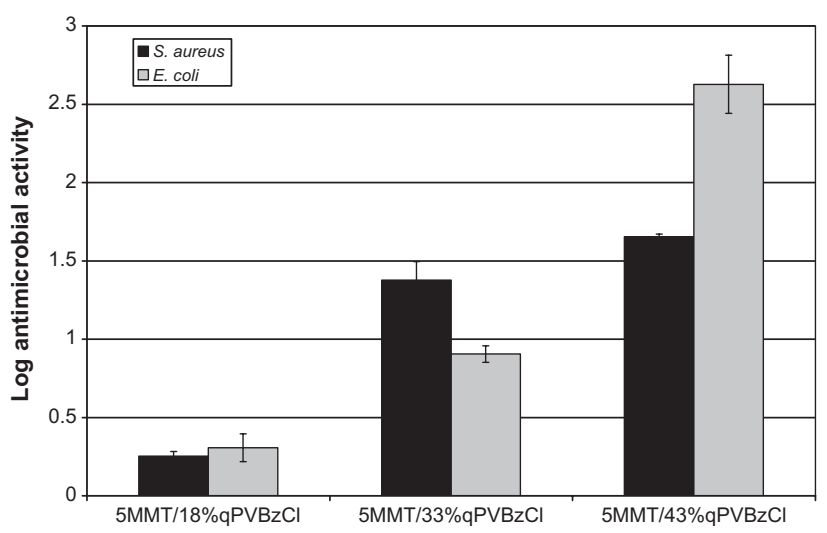

Figure 3. The dependence of the antimicrobial activity of the nanocomposites on the $\mathrm{qPVBzCl}$ content in organoclays. 
negative E. coli bacteria. The nanocomposites are effective against both bacterial strains. As anticipated, the higher qPVBzCl loading in the organoclay tends to result in better antimicrobial activity.

In many papers published so far on polymeric onium salts active by contact, the authors chose a cautious statement that the mechanism of antimicrobial action of immobilized cationic polymers has not been fully understood. In the same time, hypothesizing the antimicrobial mechanism of solid surfaces involving polymeric onium salts, is practically unequivocal and supported by experimental evidences. Generally, the proposed mechanism of antimicrobial activity of solid surfaces with immobilized quaternary onium groups was stemmed from the widely accepted action of soluble quaternary onium compounds as membrane-bursting agents. Soluble substances containing quaternary groups are adsorbed by microbial cell surface and subsequently distort cell membrane via the penetration of long alkyl chain into the membrane bi-layer structure due to hydrophobic interactions. ${ }^{[12]}$ It is widely assumed that, similar to soluble cationic polymeric biocides, surfaces with immobilized cationic polymers induce distortion of the cell membranes. Various experimental evidences have been acquired in supporting this mechanism. These included the demonstration of morphological changes of the microbial cells on the cationic surfaces and the infringement of the integrity of cellular membranes. ${ }^{[9,17]}$ Positive charge, the presence of hydrophobic long chains as a substitute in a quaternary onium group and sufficient length of polymer chains are postulated as crucial factors for this type of biocide molecule, to induce the death of cells adhered to the surface. The qPVBzCl used in this study is designed to meet these requirements. Therefore, it appears that the surfaces of the developed nanocomposites affect the bacteria in a similar way.

One of the complications of self-sterilizing surfaces of this type of material is the possible deposition of cell debris, which can potentially block cationic centers. ${ }^{[33,34]}$ In order to clarify this effect on the material developed in this study, polyamide 6 nanocomposites produced from polymerically modified organoclays were tested in four consecutive runs. The JIS Z 2801: 2000 requires the re-suspension of bacteria adhered to the surface for the subsequent viable cell counting. No additional actions were undertaken to clean the surface between the consecutive tests of the antimicrobial activity. After the bacteria re-suspension for cell counting, the samples were inoculated with a freshly prepared bacterial suspension. Figure 4 shows the antimicrobial activity of all polyamide 6 nanocomposites against $S$. aureus in the repeated tests. It can be seen that repetitive bacteria inoculation did not deteriorate the surface antimicrobial activity. There is no loss of the antimicrobial activity in repeated tests. We also conducted

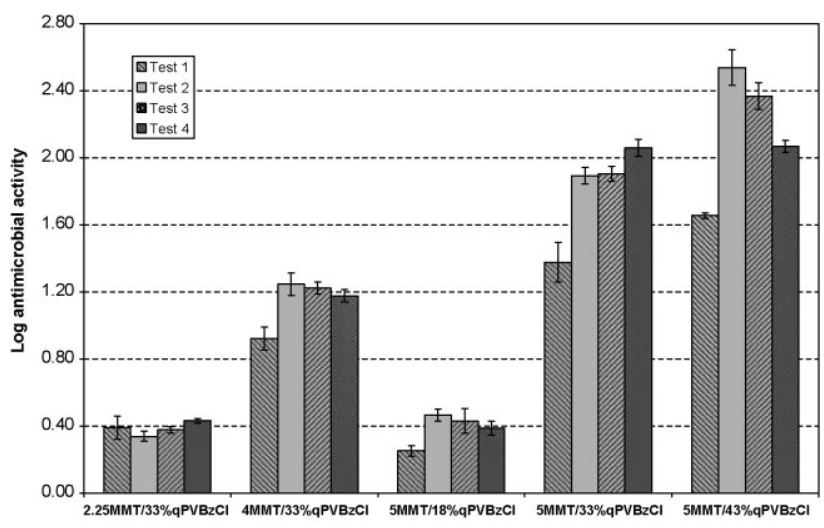

Figure 4. The stability of the antimicrobial activity of polyamide 6 nanocomposites against $S$. aureus in repeated tests.

the inhibition zone test and measurements of the electric conductivity of water with submerged nanocomposite samples as described in a previous publication. ${ }^{[20]}$ Additionally aqueous supernatants after contact with nanocomposite samples were 20-fold concentrated by ultrafiltration using membranes with molecular weight cut-off $5 \mathrm{kDa}$. Microorganism growth was studied in these solutions and no cell inhibition was observed. The results showed no migration of polymeric antimicrobial agent from the nanocomposites. The nanocomposites developed are truly non-leaching type.

Apparently as for any heterogeneous process, the reduction of the number of viable cells on the nanocomposite surface depends on contact time. According to the JIS Z 2801:2000, seeded materials are incubated for $24 \mathrm{~h}$ before counting the viable cells. In order to elucidate how quick the bacteria can be affected by the surface, we varied the incubation time of the seeded nanocomposite samples containing 5 wt.-\% of organoclays. The changes in the antimicrobial activity depending on incubation time of the bacteria, dispensed on the surface of the nanocomposites,

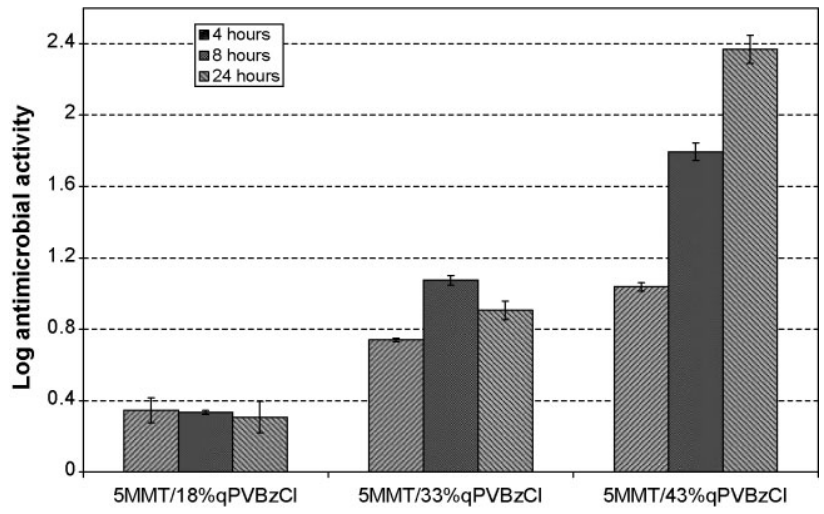

Figure 5. The influence of contact time on the antimicrobial activity of polyamide 6 nanocomposites against $E$. coli in repeated tests. 
are shown in Figure 5. Although the viability of bacterial cell on the nanocomposite surface decreased within $24 \mathrm{~h}$, substantial numbers of bacteria were killed in shorter contact periods. Interestingly, for the nanocomposites based on the organoclay containing 18 wt.- $\%$ of $\mathrm{qPVBzCl}$, the reduction in number of viable cells, did not practically improve with the increase in contact time. This suggests that for nanocomposites prepared with this organoclay, there are inactive patches on the material surface, where adhered cells can survive independently of the contact time. For this nanocomposite, antimicrobial patches do not cover the entire material surface. However, for the nanocomposites with organoclays containing more $\mathrm{qPVBzCl}$, the distribution of antimicrobial patches across the material surface, substantially excludes areas where bacteria can avoid contact with the antimicrobial polymer. Prolonged contacts are necessary to affect those bacteria, which were not in contact with the surface after the inoculation.

\section{Mechanical Properties of Antimicrobial Polyamide 6 Nanocomposites}

The progress in polymer/clay nanocomposites was predominantly driven by the ability of nanotechnology to improve mechanical properties of polymer materials. As can be seen from Table 2, tensile properties of polyamide 6 are changed with the introduction of polymerically modified organoclays. One-way analysis of variance (ANOVA) indicated that mean values of both tensile strength and elongation at break are significantly different at a $1 \%$ level of significance. The least significant difference at the $5 \%$ level was $4 \mathrm{MPa}$ and $42 \%$ for yield strength and elongation at break, respectively.

The introduction of $2.25 \mathrm{wt} . \%$ of macro-intercalated organoclay improved the yield strength by $35.5 \%$ with further improvement up to $44.6 \%$ for the nanocomposite containing 5 wt.-\% organoclays. These results suggest a good level of organoclay dispersion leading to the polymer reinforcement by particles with high aspect ratio. Accord-

Table 2. Tensile strength for polyamide 6 and its nanocomposites.

\begin{tabular}{|c|c|c|}
\hline \multirow[t]{2}{*}{ Material } & $\begin{array}{c}\text { Yield } \\
\text { strength }\end{array}$ & $\begin{array}{c}\text { Elongation } \\
\text { at break }\end{array}$ \\
\hline & $\mathrm{MPa}$ & $\%$ \\
\hline Polyamide 6 & $50.4 \pm 2.4$ & $390 \pm 71$ \\
\hline $5 \mathrm{MMT} / 18 \mathrm{qPVBzCl}$ & $73.1 \pm 1.4$ & $28.8 \pm 3.6$ \\
\hline $5 \mathrm{MMT} / 33 \mathrm{qPVBzCl}$ & $72.9 \pm 1.5$ & $29.8 \pm 3.0$ \\
\hline 4MMT/33qPVBzCl & $69.3 \pm 1.0$ & $41.2 \pm 5.4$ \\
\hline 2.25MMT/33qPVBzCl & $68.3 \pm 2.9$ & $77.1 \pm 17.3$ \\
\hline 5MMT/43qPVBzCl & $49.2 \pm 5.4$ & $18.3 \pm 2.6$ \\
\hline
\end{tabular}

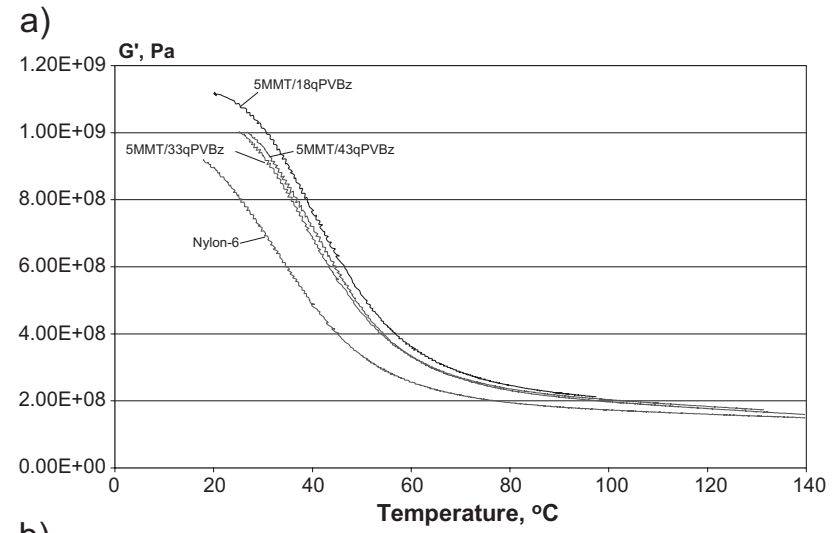

b)

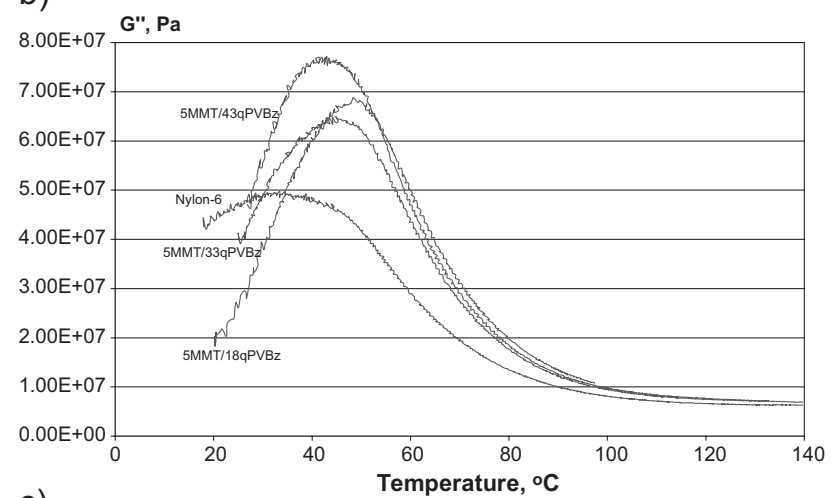

c)

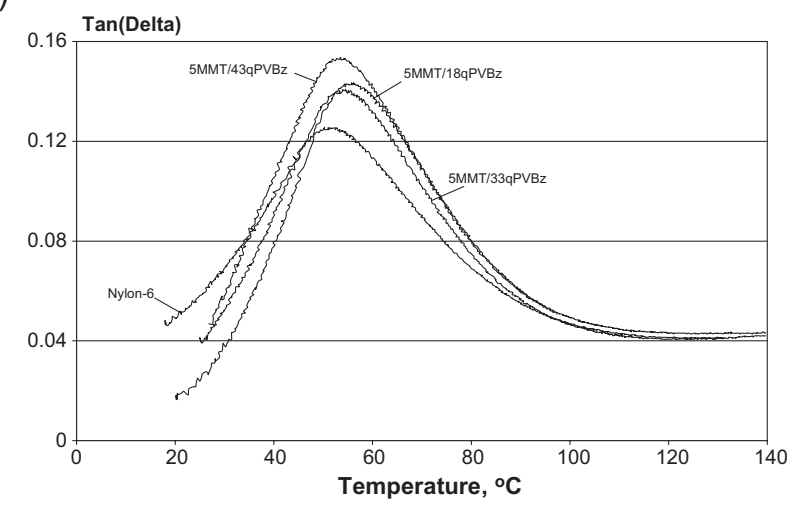

Figure 6. Temperature dependence of the storage modulus, $G^{\prime}(a)$, the loss modulus, $G^{\prime \prime}(\mathrm{b})$, and $\tan \delta(\mathrm{c})$, for the pristine polyamide 6 and antimicrobial polyamide nanocomposites.

ingly, restraining the segmental motion of the polyamide chains by the intercalated/exfoliated clay platelets causes the decrease in the elongation at break. Typically, antimicrobial nanocomposites were less ductile than polyamide 6 with more than 5 -fold decrease in values of elongation at break.

The reinforcing effect for antimicrobial nanocomposites was also confirmed by DMA tests, which showed the increase in storage modulus $\left(G^{\prime}\right)$ over the temperature range from 20 to $140^{\circ} \mathrm{C}$ [Figure 6(a)]. In the series of antimicrobial nanocomposites containing $5 \mathrm{wt.}$. $\%$ of organoclays with different amount of intercalated $\mathrm{qPVBzCl}$, the use of 
organoclay containing $18 \%$ of cationic polymer resulted in the nanocomposite with the highest mechanical strength. In accordance with the results of tensile tests, this nanocomposite has the highest values of the storage modulus. Interestingly, nanocomposites based on the organoclays with 33 and $43 \%$ content of cationic polymer showed only slight difference in the storage modulus, despite having significantly different yield strength. Some implications regarding these discrepancies might be obtainable, analyzing other parameters of the DMA data. As can be seen from Figure 6, the variations in loss $\left(G^{\prime \prime}\right)$ modulus and $\tan \delta$, indicate significant difference in viscoelastic responses of the antimicrobial nanocomposites depending on the organoclays used for composite preparation. The values of $\tan \delta$ for all antimicrobial nanocomposites are significantly higher than that for the pristine polyamide 6 . The increased viscous component may give the materials better impact resistance. Moreover, the tan $\delta$ peak shifts towards higher temperature. The highest glass transition point $\left(T_{\mathrm{g}}\right)$ was observed for nanocomposites based on organoclay containing $18 \% \mathrm{qPVBzCl}$, which is the strongest nanocomposite. The increase in $T_{\mathrm{g}}$ and tensile strength is indicative of good adhesion between the matrix polymer and clay platelets, which restricts the segmental motion of polyamide at the organic-inorganic interface.

Clay surface is progressively covered with cationic polymer with the increase in $\mathrm{qPVBzCl}$ content in organoclays. Since $T_{\mathrm{g}}$ is smaller for the nanocomposites containing organoclays with 33 and $43 \%$ of qPVBzCl, it appears that adhesion between polyamide and clay platelets deteriorates when more cationic polymers are bound to the clay surface. This suggests that, similar to the polyamide nanocomposites based on organoclays modified with cationic surfactants, ${ }^{[35]}$ polyamide 6 has higher affinity to clay surface than to qPVBzCl. Thus, mechanical strength is dependent on both the ability of polyamide to intercalate into the organoclay gallery and the availability of unblocked clay surface. The organoclay containing 18\% of the polymeric modifier, has sufficient layer expansion to assist polyamides intercalating into the organoclay. At the same time, for this organoclay, there is enough free clay surface for efficient polyamide/MMT interactions, which results in high mechanical strength. Also lower content of relative low-molecular-weight $\mathrm{qPVBzCl}$ may result in the highest tensile strength. For the composite with the qPVBzCl content of $33 \%$, the area of unoccupied surface is still sufficient to reach high yield strength. However, when organoclay contains $43 \%$ of polymeric modifier, probably qPVBzCl shields the clay surface and mechanical strength decreases abruptly. The relative lower molecular weight of qPVBzCl could also play an important role.

Apparently, antimicrobial nanocomposites are stiffer materials than pristine polyamide 6 . Nevertheless, the nanocomposites are characterized by higher values of viscous modulus compared with pristine polyamide 6 [Figure 6(b)]. It is noteworthy that such observations have been reported for polymer/clay nanocomposites. ${ }^{[36]} \mathrm{How}^{-}$ ever, there are restricted comments on such an increase in viscous component for generally stiffer materials. The formation of an interphase was suggested as a possible reason for such behavior. ${ }^{[37]}$ An interphase has been defined as the matrix material close to the clay surface, whose properties are different from those of the bulk material. It appears that for polymerically modified organoclays, the formation of the interface is very likely due to the interactions between the polymer modifier and matrix polymer. Because of a significant size of polymer modifier, the interphase could extend further from the silicate surface, compared with surfactant-modified organoclays. For the nanocomposites with interface formation, the contribution from the interphase, composed of segments of matrix polymer and modifier, to the viscous response of the nanocomposites, can be enhanced as observed for antimicrobial nanocomposites developed in this study.

One might consider that interphase formation would indicate some affinity between $\mathrm{qPVBzCl}$ and polyamide 6 . Therefore, organoclays with larger amounts of bound cationic polymers, involves more matrix polymer in the formation of interphase, and accordingly the volume fraction of interphase grows. As a result, the magnitude of the loss modulus below $T_{\mathrm{g}}$, steadily increased when organoclays with higher $\mathrm{qPVBzCl}$ were used for the nanocomposite preparation [Figure 6(b)]. Probably both the mechanical properties of the interface and the relative low molecular weight of qPVBzCl, do not improve tensile strength; so the nanocomposites based on organoclay with $43 \%$ of $\mathrm{qPVBzCl}$, which has the largest interphase and qPVBzCl volume, showed significant decrease in yield strength.

Although the results on the mechanical properties of antimicrobial nanocomposites are promising, it is expected that with polymer modifiers it is possible to reach even better mechanical properties through tailoring the molecular composition of the polymeric modifier. The technology is highly flexible in controlling antimicrobial and mechanical properties and would create a better solution to produce antimicrobial materials.

\section{Conclusion}

A recent feasibility study ${ }^{[20]}$ on clay/polymer nanotechnology in the design of the antimicrobial materials was further widened into the exploration of polymerically modified organoclays in the development of non-leaching antimicrobial polymer composites. The use of a copolymer containing quaternary ammonium groups prepared by partial amination of $\mathrm{PVBzCl}$, effectively changed MMT 
lamellar morphology and produced macromolecule-intercalated organoclays. For the polymerically modified clays, the change in the amount of bound polymer allows altering both organoclay morphology and the content of functional groups, which imparts antimicrobial activity to the materials. The interlayer spacing of the organoclays with the intercalated $\mathrm{qPVBzCl}$ was comparable with the values reported for commercially available organoclays.

X-ray diffractograms of polyamide 6 composites with macro-intercalated clays, confirmed polyamide intercalation into the gallery of organoclays. The introduction of 2.25 wt.-\% of polymerically modified organoclay, improved the yield strength by $35.5 \%$ with further improvement up to $44.6 \%$ for nanocomposite containing 5 wt.-\% of organoclays. The XRD results and mechanical properties of the prepared polyamide composites, gave indications of the dispersion of polymerically modified clay platelets in polyamide 6 matrix. The reinforcing effect for polyamide nanocomposites was also confirmed in DMA tests, which showed the increase in storage modulus $\left(G^{\prime}\right)$, meanwhile the loss modulus $G^{\prime}$ and $\tan \delta$ for antimicrobial nanocomposites, are higher than that of pristine polyamide 6 . This is important in developing strong and impact resisting materials.

Developed polyamide nanocomposites demonstrated up to 2 log reduction in the viable cells adhered to the material surface. Nanocomposites were active against both grampositive S. aureus and gram-negative E. coli bacteria, through killing bacteria on contact. After dispersion in a polymer matrix, clay platelets functionalized with cationic polymer, effectively render the surface antimicrobial properties. Moreover, there was no migration of the polymeric antimicrobial agent from the nanocomposites. Testing the antimicrobial activity of the nanocomposites in consecutive runs showed that repeated bacteria inoculation did not deteriorate surface antimicrobial activity. This work is the first to demonstrate the application of polymer/ clay nanotechnology for the production of nanocomposites with permanent, non-leaching antimicrobial surfaces.

The content of the polymeric antimicrobial agent in nanocomposites, can be varied by the change in organoclay loading, or the use of organoclays with different amounts of bound antimicrobial agent. These parameters control both antimicrobial behavior and the mechanical properties of the nanocomposites. Therefore, the optimization of the material composition is required to maximize antimicrobial efficiency and improve other engineering properties. The described approach provides a robust alternative to surface modification or coating technologies in the production of self-sterilizing surfaces.

Received: June 17, 2009; Revised: August 18, 2009; Published online: XXX; DOI: 10.1002/mame.200900166
Keywords: antimicrobial nanocomposites; organoclays; polymeric materials; polymers

[1] A. E. Madkour, G. N. Tew, Polym. Int. 2008, 57, 6.

[2] E. M. Hetrick, M. H. Schoenfisch, Chem. Soc. Rev. 2006, 35, 780.

[3] P. Appendini, J. H. Hotchkiss, Innov. Food Sci. Emerg. 2002, 3, 113.

[4] G. J. Gabriel, A. Som, A. E. Madkour, T. Eren, G. N. Tew, Mater. Sci. Eng. 2007, R57, 28.

[5] K. Lewis, A. M. Klibanov, Trends Biotechnol. 2005, 23, 343.

[6] A. J. Isquith, E. A. Abbott, P. A. Walters, Appl. Microbiol. 1972, $24,859$.

[7] J. L. Speier, J. R. Malek, J. Colloid Interface Sci. 1982, 89, 68.

[8] A. Kanazawa, T. Ikeda, T. Endo, J. Appl. Polym. Sci. 1994, 54, 1305.

[9] J. C. Tiller, C.-J. Liao, K. Lewis, A. M. Klibanov, PNAS 2001, 98 , 5981.

[10] L. Cen, K. G. Neoh, L. Ying, E. T. Kang, Surf. Interface Anal. 2004, 36,716 .

[11] J. Huang, H. Murata, R. R. Koepsel, A. J. Russell, Biomacromolecules 2007, 8, 1396.

[12] W. B. Hugo, A. D. Russell, "Types of antimicrobial agents", in: "Principles and Practice of Disinfection, Preservation and Sterilization", $3^{\text {rd }}$ edition, A. D. Russell, W. B. Hugo, G. A. J. Ayliffe, Eds., Blackwell Science, Oxford 1999, p. 5.

[13] N. Kawabata, M. Nishiguchi, Appl. Environ. Microbiol. 1988, 54, 2532.

[14] P. R. Murray, A. C. Niles, R. L. Heeren, J. Clin. Microbiol. 1988, 26, 1884.

[15] J. Hazziza-Laskar, N. Nurdin, G. Helary, G. Sauvet, J. Appl. Polym. Sci. 1993, 50, 651.

[16] Y. Nakagawa, Y. Yamano, T. Tawaratani, H. Kourai, T. Horie, I. Shibasaki, Appl. Environ. Microbiol. 1982, 43, 1041.

[17] J. Lin, J. C. Tiller, S. B. Lee, K. Lewis, A. M. Klibanov, Biotechnol. Lett. 2002, 24, 801.

[18] F. Gao, Mater. Today 2004, 7, 50.

[19] D. R. Paul, L. M. Robeson, Polymer 2008, 49, 3187.

[20] R. Nigmatullin, F. Gao, V. Konovalova, J. Mater. Sci. 2008, 43, 5728.

[21] K. E. Styan, D. J. Martin, L. A. Poole-Warren, J. Biomed. Mater. Res. A 2008, 86A, 571.

[22] K. Styan, M. Abrahamian, E. Hume, L. A. Poole-Warren, Key Eng. Mater. 2007, 342-343, 757.

[23] J.-W. Rhim, S.-I. Hong, C.-S. Ha, LWT - Food Sci. Technol. 2009, $42,612$.

[24] R. Sothornvit, J.-W. Rhim, S.-I. Hong, J. Food Eng. 2008, 78, 855.

[25] J.-W. Rhim, S.-I. Hong, H.-M. Park, P. K. W. Ng, Agric. Food Chem. 2006, 54, 5814

[26] S.-I. Hong, J.-W. Rhim, J. Nanosci. Nanotechnol. 2008, 8, 5818.

[27] J. Zhang, E. Manias, C. A. Wilkie, J. Nanosci. Nanotechnol. 2008, 8, 1597.

[28] S. Șen, N. Nugay, T. Nugay, Polym. Int. 2006, 55, 552.

[29] L. Zhu, R. Zhu, L. Xu, X. Ruan, Colloid Surf. A - Physicochem. Eng. Asp. 2007, 304, 41.

[30] X. Zheng, C. A. Wilkie, Polym. Degrad. Stabil. 2003, 81, 539.

[31] M. Sepehr, L. A. Utracki, X. Zheng, C. A. Wilkie, Polymer 2005, 46, 11557. 
[32] R. S. Nohr, J. G. MacDonald, J. Biomater. Sci. Polymer. Edn. 1994, 5, 607.

[33] Z. Cheng, X. Zhu, Z. L. Shi, K. G. Neoh, E. T. Kang, Ind. Eng. Chem. Res. 2005, 44, 7098.

[34] S. B. Lee, R. R. Koepsel, S. W. Morley, K. Matyjaszewski, Y. Sun, A. J. Russell, Biomacromolecules 2004, 5, 877.
[35] T. D. Fornes, D. L. Hunter, D. R. Paul, Macromolecules 2004, 37 1793.

[36] S. Pavlidou, C. D. Papaspyrides, Prog. Polym. Sci. 2008, 33, 1119.

[37] R. S. Fretig, III, M. R. Garnich, Compos. Sci. Technol. 2004, 64, 2577. 